\title{
Effects of some humic substance(s) based plant activators on growth, yield and quality parameters of cherry tomato cultivar under regular and stressed soil conditions
}

\author{
Turan M. ${ }^{1}$, Yıldırım E. ${ }^{2}$, Ekinci M. ${ }^{2}$, Kılıç M. ${ }^{1}$, Bolouri P. ${ }^{1}$, Hijazi N. ${ }^{1}$ \\ ${ }^{1}$ University of Yeditepe, İstanbul, Turkey, m_turan25@hotmail.com \\ ${ }^{2}$ University of Atatürk, Erzurum, Turkey \\ Keywords: plant activator, cherry tomato, growth, yield, mineral content, stressed soil \\ doi: 10.36291/HIT.2019.turan.064
}

In this study, the effects of some plant activators on plant growth characteristics, yield and biochemical properties of cherry tomatoes grown in two different soil media (stressed and regular soil) were investigated under controlled greenhouse conditions. Studies were conducted in pots. Powhumus (PH), seed covering (SC), root enhancer (RE) and fulvic acid (FA) were treated as seed and drench applications. Treatments were grouped as follows: Control 0: no treatments, $\mathrm{PH}$ 1: $\mathrm{PH}$ seed coating, SC 1: SC seed coating, $\mathrm{PH} 2 \mathrm{PH}$ seed coating $+\mathrm{PH}, \mathrm{SC} 2$ : SC seed coating $+\mathrm{PH}$, RE: RE seed coating +RE, FA: FA seed coating +FA. In order to determine the effects of the applications on plant growth parameters were examined, such as dry plant and root weight, plant height, stem diameter, leaf number; yield parameters such as fruit number per cluster, fruit height, fruit diameter, fruit weight, cluster weight, cluster number per plant and yield per plant; mineral content of leaf, root and fruit; chlorophyll reading value (SPAD); and some fruit characteristics such as total soluble solids (TSS), fruit dry matter (DM), and vitamin C. Furthermore, some physiological and biochemical characteristics of tomato leaf such as malondialdehyde (MDA), hydrogen peroxide $\left(\mathrm{H}_{2} \mathrm{O}_{2}\right)$, proline and sucrose, and antioxidant enzyme activities (catalase-CAT, peroxidase-POD and superoxide dismutase-SOD) were also determined. All plant activators improved plant growth and yield of tomato under both soil conditions. Plant activator applications increased the plant height, stem diameter, leaf number, SPAD, plant dry weight and root dry weight, and the highest effect were determined as 59\% (FA), 47\% (RE), 54\% (SC2), 38\% (FA), 60\% (RE) and 70\% (RE) in regular soil, and as 79\% (FA), 50\%(RE), 60\%(FA), 42\%(FA), 78\%(FA), and 72\% (FA) in stressed soil, respectively, as compared to the control 0 treatment. With the applications, increases in DM, vitamin C, TSS, fruit height, fruit diameter, fruit weight, cluster weight, cluster number per plant, fruit per cluster, yield per plant were 12\%(RE), 33\% (SC1), 29\% (SC1), 15\% (SC2), 23\% (SC2), 43\% (SC2), 45\% (SC2), 21\% (RE), 26\% (SC1) and 56\% (RE) in regular soil and 41\% (SC2), 40\% (SC1), 38\% (SC1), 21\% (FA), 26\% (FA), 53\% (FA), 54\% (FA), 58\% (FA), 58\% (FA) and $81 \%$ (FA) in stressed soil, respectively, as compared to the control 0 . According to the study result, FA generally had more positive effects than the other treatments under stressed soil conditions. Applications were more effective on plant growth, fruit characters and yield parameters in stressed soil plants than those in regular soil. RE was the most effective application for mineral content (leaf, root and fruit) in regular soil, whereas the most effective application in stressed soil was FA. N, $\mathrm{P}, \mathrm{K}, \mathrm{Ca}, \mathrm{Mg}, \mathrm{Mn}, \mathrm{Na}, \mathrm{Zn}, \mathrm{B}, \mathrm{Fe}$ and $\mathrm{Cl}$ content (leaf, root and fruit) in regular soil was increased by $23-90 \%$ as compared to control, whereas this was ranged from 40 to $92 \%$ in stressed soil. CAT, POD and SOD activity, $\mathrm{H}_{2} \mathrm{O}_{2}, \mathrm{MDA}$ and proline content were decreased in regular and stressed soil with treatments as compared to control, but POD activity was more in SC 1 application in stressed soil. The content of sucrose elevated with especially RE treatment ( $57 \%$ increase in regular soil, $65 \%$ increase in stressed compared to control). In conclusion, plant activator treatments could be suggested to be used especially in cherry tomato cultivation in problematic soil areas. 